\title{
Optimal Licensing in a Stackelberg Duopoly Market under Asymmetric Information of the Marginal Cost
}

\author{
Qingyou Yan ${ }^{\mathrm{a}, \mathrm{b}}$, Le Yang ${ }^{\mathrm{a}, *}$, Jieting Yin ${ }^{\mathrm{a}}$, Youwei Wan ${ }^{\mathrm{a}}$ \\ ${ }^{a}$ School of Economic and Management, North China Electric Power University, Beijing, 102206, China \\ ${ }^{b}$ Beijing Energy Development Research Center, Beijing, 102206, China
}

\begin{abstract}
In this paper, we investigate a Stackelberg leader's licensing behavior and its welfare consequence when the rival holds private information about the marginal cost after licensing occurs. In order to examine the effect of the asymmetric information on the optimal licensing strategy, we consider three possible forms of a two-part tariff licensing contract (excluding excluding contract, separating contract, and pooling contract). The result shows that, the optimum is either an exclusive contract with pure royalty on the low type rival or a separating contract with different royalty rates on the different type rivals, which mainly depends on the possibility that the rival is a low type. Furthermore, there is a conflict between the innovator and social welfare when the possibility that the rival is a low type is very high.
\end{abstract}

Keywords: technology licensing; stackelberg competition; asymmetric information; two-part tariff; social welfare

(Submitted on November 13, 2017; Revised on December 21, 2017; Accepted on January 19, 2018)

(C) 2018 Totem Publisher, Inc. All rights reserved.

\section{Introduction}

Licensing has been viewed as a quick and effective method to disseminate technology innovations. It does not transfer the ownership of licensed technology but merely gives licensee the right to use the technology. A key issue is how to develop a scheme to transfer this right to improve innovator's payoffs, incentives and capabilities. A number of previous research has tried to explore the optimal choice for the innovator under different market structures, given the options of fixed-fee, royalty or two-part tariff licensing (see Wang [11,12]; Sen and Tauman [10]; Li and Yanagawa [5]; Kishimoto and Muto [6]; Bagchi and Mukherjee [1]; Chen et al. [2]; Yan and Yang [13]). However, a critical assumption made in many existing studies (including the above-mentioned ones) is that the innovator has complete information about its rival's cost and thus designs the corresponding licensing contracts. This may be unrealistic.

In the real world, both the confidentiality of production process and the uncertainty of the application of new technology generally allow the licensee to hold some private information before and/or after technology licensing. In this situation, due to the licensor's limited control over the licensee, it is difficult for both parties to reach an agreement over the value of a technology innovation. Thus, an optimal licensing contract should be designed to prevent the risk of reverse selection or moral hazard caused by this kind of asymmetric information. Gallini and Wright [3], Poddar and Sinha [7] and Schmitz [8], all show that a royalty licensing contract can effectively handle the reverse selection problems. Sen [9] argues that the asymmetric information regarding the licensee's costs leads to diversification in optimal licensing mechanisms.

These studies concerning on technology licensing under asymmetric information are routine from the perspective of an outside innovator. In fact, many technology innovators are also the producer in the market. They care about not only the licensing revenue, but also the influence of licensing the new technology on their advantage positions in the competition. Hence, compared to the outside innovators, the inside innovators will face more complicated incentives and have to choose

\footnotetext{
* Corresponding author.

E-mail address: ncepu_yl@126.com
} 
the different licensing mechanisms when they intend to transfer their new technology. Heywood et al. [4] recently explored the insider's optimal two-part tariff licensing contract when it holds imperfect information about the value of the patent. However, they carry out the research based on the assumption that the two firms are simultaneous-move. What will happen if the innovative firm has the first move advantage? Hence, it is necessary to consider this issue in a leadership structure.

In this paper, we expand the consideration of asymmetric information to the licensing decision for an inside innovator who serves as a market leader in a Stackelberg competition. Different from some literature focusing on asymmetric information before licensing occurs, we consider the case in which the rival has some private information about the marginal cost after it obtains the innovation. Due to this asymmetric information, three possible forms of a two-part tariff licensing contract will be examined. The aim is to analyze the effect of the diversification of licensing contract on the optimal strategy and thus what kind of licensing contract will be adopted by the leading firm. Furthermore, the consequence of choosing the optimal licensing contract on social welfare will also be examined to see whether there is a consistency (or conflict) between the innovator and the whole society.

The outline of this study is as follows. In Section 2, we describe the basic model and derive the pre-licensing equilibrium outcomes for the two firms. Section 3 investigates three possible form of a two-part tariff licensing contract. Section 4 compares the three above-mentioned forms of licensing contract. In Section 5, we offer the conclusions.

\section{The basic model and the no-licensing case}

Consider a Stackelberg model with two firms, the leader (firm 1) and the follower (firm 2). Only the leader undertakes technology innovation and may license it to the follower. Prior to technology licensing, the leader and follower's initial marginal costs are respectively 0 and $\tilde{c}$. If the follower obtains the innovation, then its marginal cost will become private information and the leader will only has a prior belief about it. With the probability $\theta$, the leader believes that the follower's marginal cost is 0 (the low type, referred to as " $l$ ") and with the probability $(1-\theta)$, the leader believes the follower's marginal cost is $c(<\tilde{c}$ ) (the high type, referred to as " $h$ "). Here, $\theta$ is the common knowledge to both firms.

The whole game comprises of two stages. In the first stage, the leader offers a two-part tariff contract (the combination of fixed-fee and per-unit royalty) to the follower, and the follower decides whether or not to accept the contract. If the follower accepts the contract, it will pay the licensing fee according to the contract and the marginal cost will be reduced; if the follower does not, nothing will occur. In the second stage, given the technological configuration at the end of the first stage, the two firms choose their outputs sequentially and compete in the market. The whole game will be solved by backward induction.

The linear inverse demand function is assumed by $p=a-q_{1}-q_{2}$, where $p$ is the market price, and $q_{1}$ and $q_{2}$ are the outputs of firms 1 and 2, respectively. If the two firms' marginal costs are given by $c_{1}$ and $c_{2}$, then in the competition stage, the Stackelberg equilibrium quantities for the two firms are

$$
q_{1}\left(c_{1}, c_{2}\right)=\frac{\left(a-2 c_{1}+c_{2}\right)}{2}, \quad q_{2}\left(c_{1}, c_{2}\right)=\frac{\left(a+2 c_{1}-3 c_{2}\right)}{4} .
$$

and the corresponding payoffs are

$$
\pi_{1}\left(c_{1}, c_{2}\right)=\frac{\left(a-2 c_{1}+c_{2}\right)^{2}}{8}, \quad \pi_{2}\left(c_{1}, c_{2}\right)=\frac{\left(a+2 c_{1}-3 c_{2}\right)^{2}}{16}
$$

Now, we first consider the no-licensing case. Substitute $\left(c_{1}, c_{2}\right)=(0, \tilde{c})$ into Equations (2), we have

$$
\pi_{1}^{N L}=\frac{(a+\tilde{c})^{2}}{8}, \quad \pi_{2}^{N L}=\frac{(a-3 \tilde{c})^{2}}{16}
$$


where the superscript " $N L$ " denotes "no-licensing." The innovation is drastic if $\tilde{c}>a / 3$; the follower is driven out of the market $\left(q_{2}^{N L}<0\right)$ and the leader becomes a monopolist. Therefore, we only focus on the general and more interesting case where the innovation is non-drastic and assume that $\tilde{c}<a / 3$ throughout the whole study.

We also assume that social welfare is defined as the sum of the two firms' profits and consumer surplus. Hence, in the no-licensing case, it follows

$$
W^{N L}=\pi_{1}^{N L}+\pi_{2}^{N L}+\frac{1}{2}\left(q_{1}^{N L}+q_{2}^{N L}\right)^{2}=\frac{1}{8}(a+\tilde{c})^{2}+\frac{1}{16}(a-3 \tilde{c})^{2}+\frac{1}{32}(3 a-\tilde{c})
$$

\section{The licensing case}

In this section, we analyze a two-part tariff licensing contract and its implication for social welfare. Due to the uncertainty of the follower's type, the licensing contract will appear as three different forms, and the leader's total profit will become the expected profit. In order to facilitate the following discussion, we first give the general equilibrium in the competition stage.

Suppose that the two firms' marginal costs are $c_{1}$ and $c_{2}$, respectively, and the follower accepts the two-part licensing contract $(F, r)$ offered by the leader. Then, the profit functions of the two firms in the competition stage are

$$
\left\{\begin{array}{l}
\Pi_{1}=\left(a-q_{1}-q_{2}\right) q_{1}-c_{1} q_{1}+r q_{2}+F=\pi_{1}+F \\
\Pi_{2}=\left(a-q_{2}-q_{1}\right) q_{2}-c_{2} q_{2}-r q_{2}-F=\pi_{2}-F
\end{array}\right.
$$

The equilibrium results are given as follows

$$
\begin{gathered}
q_{1}\left(c_{1}, c_{2}, r\right)=\frac{1}{2}\left(a-2 c_{1}+c_{2}\right), \quad q_{2}\left(c_{1}, c_{2}, r\right)=\frac{1}{4}\left(a+2 c_{1}-3 c_{2}-2 r\right) . \\
\pi_{1}\left(c_{1}, c_{2}, r\right)=\frac{1}{8}\left(a-2 c_{1}+c_{2}\right)\left(a+2 c_{1}+c_{2}+2 r\right)+\frac{r}{4}\left(a+2 c_{1}-3 c_{2}-2 r\right), \\
\pi_{2}\left(c_{1}, c_{2}, r\right)=\frac{1}{16}\left(a+2 c_{1}-3 c_{2}-2 r\right)^{2} .
\end{gathered}
$$

\subsection{The excluding contract}

In this case, the leading firm offers a contract acceptable only to the low type rival. In order to exclude the high type one, the offered contract $\left(F^{E}, r^{E}\right)$ to both types should satisfy $r^{E}>3(\tilde{c}-c) / 2$. Then, the follower is a low type and accepts the license with the possibility of $\theta$, whereas if it is a high type, it rejects the license with the possibility of $(1-\theta)$. Hence, the leader's maximization problem is described as

$$
\begin{gathered}
\max _{\left(F^{E}, r^{E}\right)} \theta\left[\pi_{1}\left(0,0, r^{E}\right)+r^{E} q_{2}\left(0,0, r^{E}\right)+F^{E}\right]+(1-\theta) \pi_{1}^{N L} \\
\text { s.t. } \pi_{2}\left(0,0, r^{E}\right)-F^{E} \geq \pi_{2}^{N L}, \frac{3}{2}(\tilde{c}-c) \leq r^{E} \leq \frac{3}{2} \tilde{c} .
\end{gathered}
$$

The participation constraints (9) ensure that the low type follower will accept the contract but the high type follower won't. The maximum fixed-fee that can be charged by the leader is $F^{E}=\pi_{2}\left(0,0, r^{E}\right)-\pi_{2}^{N L}$. Substitute it into Eq. (8), we have

$$
\Pi_{1}^{E}\left(r^{E}\right)=\theta\left[\pi_{1}\left(0,0, r^{E}\right)+r^{E} q_{2}\left(0,0, r^{E}\right)+\pi_{2}\left(0,0, r^{E}\right)-\pi_{2}^{N L}\right]+(1-\theta) \pi_{1}^{N L}
$$


Since $\partial \Pi_{1}^{E} / \partial r^{E}=\left(a-2 r^{E}\right) / 4>0$, we can directly deduce that the optimal royalty rate is $r^{E}=3 \tilde{c} / 2$, and thus the fixedfee is $F^{E}=0$. Then, the expected profit for the leader is

$$
\Pi_{1}^{E}=\frac{1}{8}(a+\tilde{c})^{2}+\frac{1}{2} \theta \tilde{c}\left(a-\frac{5}{2} \tilde{c}\right)
$$

Since $a>3 \tilde{c}$, it follows that $\Pi_{1}^{E}>\pi_{1}^{N L}$ for all $\theta \in[0,1]$. The corresponding social welfare is

$$
W^{E}=\Pi_{1}^{E}+\theta\left[\pi_{2}^{E}+\frac{1}{2}\left(q_{1}^{E}+q_{2}^{E}\right)^{2}\right]+(1-\theta)\left[\pi_{2}^{N L}+\frac{1}{2}\left(q_{1}^{N L}+q_{2}^{N L}\right)^{2}\right]=W^{N L}+\frac{1}{8} \theta \tilde{c}(a-8 \tilde{c}) .
$$

Lemma 1. Under excluding contract, the optimal licensing policy is a pure royalty contract with $r^{E}=3 \tilde{c} / 2$ for the low type follower. Thus, the leader's profit is $\Pi_{1}^{E}=\frac{1}{8}(a+\tilde{c})^{2}+\frac{1}{2} \theta \tilde{c}\left(a-\frac{5}{2} \tilde{c}\right)$.

\subsection{The separating contract}

In this case, each type of follower accepts the license by paying different prices. Note that, under this kind of licensing contract, besides the participation constraints, it also needs the incentive compatibility constraints to ensure that one type follower would not mimic the other type one in order to accept the contract, which is not in line with its own type. Suppose that the leader offers a licensing menu $\left\{\left(F_{h}^{S}, r_{h}^{S}\right),\left(F_{l}^{S}, r_{l}^{S}\right)\right\}$ to both types, the maximization problem of the leader's expected profit is described as follows

$$
\begin{aligned}
& \max _{\left.\left\{\left(F_{h}^{S}, r_{h}^{S}\right),\left(F_{l}^{S}, r_{l}^{S}\right)\right\}\right\}} \theta\left[\pi_{1}\left(0,0, r_{l}^{S}\right)+r_{l}^{S} q_{2}\left(0,0, r_{l}^{S}\right)+F_{l}^{S}\right]+(1-\theta)\left[\pi_{1}\left(0, c, r_{h}^{S}\right)+r_{h}^{S} q_{2}\left(0, c, r_{h}^{S}\right)+F_{h}^{S}\right] \\
& \qquad \begin{aligned}
\text { s.t. } & \pi_{2}\left(0,0, r_{l}^{S}\right)-F_{l}^{S} \geq \pi_{2}^{N L}, \\
& \pi_{2}\left(0, c, r_{h}^{S}\right)-F_{h}^{S} \geq \pi_{2}^{N L}, \\
& \pi_{2}\left(0,0, r_{l}^{S}\right)-F_{l}^{S} \geq \pi_{2}^{l h}-F_{h}^{S}, \\
& \pi_{2}\left(0, c, r_{h}^{S}\right)-F_{h}^{S} \geq \pi_{2}^{h l}-F_{l}^{S}, \\
& 0 \leq r_{l}^{S} \leq \frac{3}{2} \tilde{c}, \quad 0 \leq r_{h}^{S} \leq \frac{3}{2}(\tilde{c}-c) .
\end{aligned}
\end{aligned}
$$

Here, constraints (14) and (15) are the participation constraints to guarantee that both types of followers will accept the licensing contract; constraints (16) and (17) are the incentive compatibility constraints to ensure that the follower chooses the right contract designed for its own type, where $\pi_{2}^{\text {lh }}$ denotes the deviation payoff of the low type follower after falsely choosing $\left(F_{h}^{S}, r_{h}^{S}\right)$ and $\pi_{2}^{h l}$ denotes the deviation payoff of the high type follower after falsely choosing $\left(F_{l}^{S}, r_{l}^{S}\right)$; constraint (18) suggests the maximum royalties that the leader can set in the contract.

Before solving this maximization problem, we should first compute the follower's deviation payoff in constrains (16) and (17). Consider the case in which the low type follower pretends to be a high type one in the competition stage, the leader believes it is a high type and decides its output $q_{1}^{l h}=(a+c) / 2$. Although the follower knows that it is a low type, it still chooses the output $q_{2}^{l h}=\left(\mathrm{a}-q_{1}^{l h}-r_{h}^{S}\right) / 2=\left(a-c-2 r_{h}^{S}\right) / 4$ according to the leader's output $q_{1}^{\text {lh }}$. Thus, the market equilibrium price and the low type follower's payoff are respectively

$$
p^{l h}=\frac{1}{4}\left(a-c+2 r_{h}^{S}\right), \quad \pi_{2}^{l h}=\frac{1}{16}\left(a-c-2 r_{h}^{S}\right)^{2} .
$$


Repeat the similar exercise on the high type's deviation payoff, we have

$$
q_{1}^{h l}=\frac{1}{2} a, \quad q_{2}^{h l}=\frac{1}{4}\left(a-2 c-2 r_{l}^{S}\right), \quad p^{h l}=\frac{1}{4}\left(a+2 c+2 r_{l}^{S}\right), \quad \pi_{2}^{h l}=\frac{1}{16}\left(a-2 c-2 r_{l}^{S}\right)^{2}
$$

Due to asymmetric information after licensing occurs, we can conjecture that the low type follower has an incentive to pretend to be a high type one in order to pay a lower royalty rate and thus be more aggressive in the competition, whereas the high type follower usually accepts the right contract designed for its own type. Hence, the IC constraint for the low type and the participation constraint for the high type must be binding at the optimum. Thus, from the binding conditions (15) and (16), we have

$$
F_{h}^{S}=\frac{1}{16}\left(a-3 c-2 r_{h}^{S}\right)^{2}-\frac{1}{16}(a-3 \tilde{c})^{2}, \quad F_{l}^{S}=\frac{1}{16}\left(a-2 r_{l}^{S}\right)^{2}-\frac{1}{16}\left(a-c-2 r_{h}^{S}\right)^{2}+F_{h}^{S} .
$$

Then, the form of the maximization problem reduces as

$$
\max _{\left\{r_{l}^{S}, r_{h}^{S}\right\}} \Pi_{1}^{S}\left(r_{l}^{S}, r_{h}^{S}\right)=\theta\left[\pi_{1}\left(0,0, r_{l}^{S}\right)+r_{l}^{S} q_{2}\left(0,0, r_{l}^{S}\right)+F_{l}^{S}\right]+(1-\theta)\left[\pi_{1}\left(0, c, r_{h}^{S}\right)+r_{h}^{S} q_{2}\left(0, c, r_{h}^{S}\right)+F_{h}^{S}\right] .
$$

Differentiate $\Pi_{1}^{S}$ separately with respect to $r_{l}^{S}$ and $r_{h}^{S}$, we have

$$
\frac{\partial \Pi_{1}\left(r_{l}^{S}, r_{h}^{S}\right)}{\partial r_{l}^{S}}=\frac{1}{4} \theta\left(a-2 r_{l}^{S}\right)>0, \quad \frac{\partial \Pi_{1}\left(r_{l}^{S}, r_{h}^{S}\right)}{\partial r_{h}^{S}}=\frac{1}{2} \theta c+\frac{1-\theta}{4}\left(a+c-2 r_{h}^{S}\right)>0 .
$$

The positive first-order conditions imply that the royalty rates should be raised as more as possible. However, for the IC conditions (16) and (17) to simultaneous hold, a necessary condition is that $c+2 r_{h}^{S}-2 r_{l}^{S} \geq 0$, which gives a new upper bound on the royalty rate of the low type follower. Associated with condition (18), it follows that $r_{h}^{S}=3(\tilde{c}-c) / 2$ and $r_{l}^{S}=3 \tilde{c} / 2-c$, and the corresponding fixed-fees are $F_{h}^{S}=F_{l}^{S}=0$. Thus, the leader's expected profit is

$$
\Pi_{1}^{S}=\frac{1}{8}(a+\tilde{c})^{2}+\frac{1}{4}(\tilde{c}-c)(2 a+c-5 \tilde{c})-\frac{\theta}{4} c^{2}
$$

It is clear that $\Pi_{1}^{S}>\pi_{1}^{N L}$ for $\theta<\theta^{S}=(2 a+c-5 \tilde{c})(\tilde{c}-c) / c^{2}$. Correspondingly, social welfare is

$$
W^{S}=\Pi_{1}^{S}+\theta\left[\pi_{2 l}^{S}+\frac{1}{2}\left(q_{1}^{l}+q_{2}^{l}\right)^{2}\right]+(1-\theta)\left[\pi_{2 h}^{S}+\frac{1}{2}\left(q_{1}^{h}+q_{2}^{h}\right)^{2}\right]=W^{N L}+\frac{1}{8}(\tilde{c}-c)(a-8 \tilde{c}+c)+\frac{1}{4} \theta c(a-3 \tilde{c})
$$

Lemma 2. Under separating contract, the optimal licensing policy is a pure royalty contract with the different rates $r_{l}^{s}=(3 \tilde{c}-2 c) / 2$ and $r_{h}^{s}=3(\tilde{c}-c) / 2$ separately for the low and high type followers. And thus, the leader's profit is $\Pi_{1}^{S}=\frac{1}{8}(a+\tilde{c})^{2}+\frac{1}{4}(\tilde{c}-c)(2 a+c-5 \tilde{c})-\frac{\theta}{4} c^{2}$.

\subsection{The pooling contract}

In this case, the same licensing contract is offered to the follower, regardless of its type. Since the follower's marginal cost is still the private information and its production emerges as a random variable, the leader chooses its output only according to the follower's expected output in the competition stage. Thus, the associated profits of the two firms are

$$
\left\{\begin{array}{l}
\Pi_{1}=\left[a-q_{1}-E\left(Q_{2}\right)\right] q_{1}-c_{1} q_{1}+r E\left(Q_{2}\right)+F=\pi_{1}+F \\
E\left(\Pi_{2}\right)=\left[a-E\left(Q_{2}\right)-q_{1}\right] E\left(Q_{2}\right)-E\left(C_{2}\right) E\left(Q_{2}\right)-r E\left(Q_{2}\right)-F=E\left(\pi_{2}\right)+F
\end{array}\right.
$$


where $E\left(C_{2}\right)=(1-\theta) c$ and $E\left(Q_{2}\right)$ are the follower's expected marginal cost and output, respectively. $E\left(\Pi_{2}\right)$ is the corresponding expected profit. Solve for the equilibrium outputs, we have

$$
q_{1}^{P}\left(c_{1}\right)=\frac{1}{2}\left[a-2 c_{1}+E\left(C_{2}\right)\right], \quad E\left(Q_{2}\left(c_{1}\right)\right)=\frac{1}{4}\left[a+2 c_{1}-3 E\left(C_{2}\right)-2 r\right]
$$

Substitute the two equations into the best-response function of the follower, $q_{2}=\left(a-q_{1}-c_{2}-r\right) / 2$, we have

$$
q_{2}^{P}\left(c_{1}, c_{2}, r\right)=\frac{1}{4}\left[a-E\left(C_{2}\right)+2 c_{1}-2 c_{2}-2 r\right], \quad \pi_{2}^{P}\left(c_{1}, c_{2}, r\right)=\frac{1}{16}\left[a-E\left(C_{2}\right)+2 c_{1}-2 c_{2}-2 r\right]^{2} .
$$

Thus, the expected profit for the leader is

$$
\pi_{1}^{P}\left(c_{1}, r\right)=\frac{1}{8}\left[a-2 c_{1}+E\left(C_{2}\right)\right]\left[a+2 c_{1}+E\left(C_{2}\right)+2 r\right]+\frac{r}{4}\left[a+2 c_{1}-3 E\left(C_{2}\right)-2 r\right] .
$$

Suppose that the leader offers a pooling contract $\left(F^{P}, r^{P}\right)$, the maximization problem can be described as follows

$$
\begin{array}{cc}
\max _{\left(F^{P}, r^{P}\right)} & \pi_{1}^{P}\left(0, r^{P}\right)+r^{P} E\left(Q_{2}(0)\right)+F^{P} \\
\text { s.t. } & \pi_{2}^{P}\left(0,0, r^{P}\right)-F^{P} \geq \pi_{2}^{N L}, \\
& \pi_{2}^{P}\left(0, c, r^{P}\right)-F^{P} \geq \pi_{2}^{N L} .
\end{array}
$$

Since $\pi_{2}^{P}\left(0, c, r^{P}\right)<\pi_{2}^{P}\left(0,0, r^{P}\right)$, the optimal contract should be such that the high type's participation constraint (32) is binding. It implies that the maximum fixed fee is

$$
F^{P}=\pi_{2}\left(0, c, r^{P}\right)-\pi_{2}^{N L}=\frac{1}{16}\left[a-E\left(\mathrm{C}_{2}\right)-2 c-2 r^{P}\right]^{2}-\frac{1}{16}(a-3 \tilde{c})^{2}
$$

Substituting it into to the objective function and from the first-order condition with respect to $r^{P}$, we have the optimal royalty is $r^{P}=(3 \tilde{c} / 2)-c-\left(E\left(\mathrm{C}_{2}\right) / 2\right)=3(\tilde{c}-c) / 2+\theta c$, and the fixed fee becomes $F^{P}=0$. Thus, the expected profit for the leader is

$$
\Pi_{1}^{P}=\frac{1}{8}(a+\tilde{c})^{2}+\frac{1}{4}(\tilde{c}-c)(2 a+c-5 \tilde{c})-\frac{1}{4} \theta(2-\theta) c^{2}
$$

Obviously, it follows $\Pi_{1}^{P}>\pi_{1}^{N L}$ only if $\theta<\theta^{P}=1-\sqrt{2 c(a+c-3 \tilde{c})-\tilde{c}(2 a-5 \tilde{c})} / c$. The corresponding social welfare is

$$
\begin{aligned}
W^{P} & =\Pi_{1}^{P}+\theta\left[\pi_{2}^{P}\left(0,0, r^{P}\right)+\frac{1}{2}\left(q_{1}^{P}(0)+q_{2}^{P}\left(0,0, r^{P}\right)\right)^{2}\right]+(1-\theta)\left[\pi_{2}^{P}\left(0, c, r^{P}\right)+\frac{1}{2}\left(q_{1}^{P}(0)+q_{2}^{P}\left(0, c, r^{P}\right)\right)^{2}\right] \\
& =W^{N L}+\frac{1}{8}(\tilde{c}-c)(a-8 \tilde{c}+c)+\frac{1}{4} \theta c(a-3 \tilde{c})-\frac{1}{8} \theta(1-\theta) c^{2}
\end{aligned}
$$

Lemma 3. Under pooling contract, the optimal licensing policy is a pure royalty contract with $r^{P}=3(\tilde{c}-c) / 2+\theta c$ for the both type followers. Thus, the leader's expected profit is $\Pi_{1}^{P}=\frac{1}{8}(a+\tilde{c})^{2}+\frac{1}{4}(\tilde{c}-c)(2 a+c-5 \tilde{c})-\frac{1}{4} \theta(2-\theta) c^{2}$.

\section{The comparison results}

In above sections, we have obtained the leader's profits in each possible form of the two-part tariff licensing contract. Now, we should explore which form of the two-part tariff licensing contract is the optimal choice for the leading firm. A comparison across the leader's profits yields the following proposition. 
Proposition 1. Under asymmetric information about the rival's marginal cost after licensing, a Stackelberg leader prefers the separating contract by charging each type of rival with a different royalty if $\theta<\theta_{1}=\frac{(a-5 \tilde{c}+c)(\tilde{c}-c)}{\tilde{c}(2 a-5 \tilde{c})+c^{2}}$; otherwise, it prefers the excluding contract by charging only the low type rival with a royalty.

Proof. First note that $\Pi_{1}^{S}>\Pi_{1}^{P}$ always satisfies since $\left(\Pi_{1}^{S}-\Pi_{1}^{P}\right)$ is an opening downward para-cave for all $\theta \in[0,1]$. Next, solving the equation $\left(\Pi_{1}^{E}-\Pi_{1}^{S}\right)=0$ for $\theta$ yields the positive $\operatorname{root} \theta_{1}=\frac{(a-5 \tilde{c}+c)(\tilde{c}-c)}{\tilde{c}(2 a-5 \tilde{c})+c^{2}}<\theta^{S}$. Comparing $\Pi_{1}^{E}$ and $\Pi_{1}^{S}$, we can have the proposition immediately.

The proof is completed.

Proposition 1 illustrates that if there is a high possibility that the follower is a low type, then the leader prefers the excluding contract to extract the royalty rate as high as possible to refrain this potential strong competitor from being aggressive in the final competition. Although the leader loses any royalty revenue from the high type one under this contract, it turns out that this kind of licensing revenue is rather small when $\theta$ is high, and thus can be compensated by the low type's payments if the charged rate is raised to the maximum. In other words, when the difference between the marginal cost realizations is quite large after licensing occurs, the leader has an incentive to drop to license to both type followers, but only concentrate on the low type one.

If the leader has full information about the rival's realized marginal cost, both types would be licensed with the optimal rates $r^{l}=3 \tilde{c} / 2$ and $r^{h}=3(\tilde{c}-c) / 2$. However, due to the asymmetric information, whenever the leader wishes to license to the high type follower and adopt a separating contract, it has to concede an information rent to the low type one due to the asymmetric information, which results a lower royalty rate $r_{l}^{S}=(3 \tilde{c}-2 c) / 2$ compared to the situation wherein the leader has the full information about its rival's realized marginal cost.

Next, we focus on the effects of these possible contracts from the perspective of social welfare. Direct comparisons allow us to establish the following proposition.

Proposition 2. Social welfare associated with the excluding contract is greater than other cases only if $a-8 \tilde{c}+c<0$ and $\theta>\theta_{2}=\frac{(a-8 \tilde{c}+c)(\tilde{c}-c)}{\tilde{c}(a-8 \tilde{c})-2 c(a-3 \tilde{c})}$; otherwise, social welfare with the separating contract is always greater than other cases.

Proof. We can directly derive that $W^{S}>W^{E}$ and $W^{P}>W^{E}$ for all $\theta \in[0,1]$. As for the comparison of $W^{S}$ and $W^{P}$, Subtract these two functions, we have

$$
W^{E}-W^{S}=\left[-\frac{1}{4} c(a-3 \tilde{c})+\frac{1}{8} \tilde{c}(a-8 \tilde{c})\right] \theta-\frac{1}{8}(\tilde{c}-c)(a-8 \tilde{c}+c) .
$$

Since $c<\tilde{c}$, it follows that $-\frac{1}{4} c(a-3 \tilde{c})+\frac{1}{8} \tilde{c}(a-8 \tilde{c})<0$. In addition, if $a-8 \tilde{c}+c>0$, it has $W^{E}<W^{S}$ for all $\theta \in[0,1]$. If $a-8 \tilde{c}+c<0$, solving $W^{S}(\theta)=W^{P}(\theta)$ for $\theta$ yields the root $\theta_{2}=\frac{(a-8 \tilde{c}+c)(\tilde{c}-c)}{\tilde{c}(a-8 \tilde{c})-2 c(a-3 \tilde{c})}$, and we obtain that $W^{P}>W^{S}$ only if $\theta>\theta_{2}$

The proof is completed.

Proportion 2 shows that there is a conflict between the leader's profit maximization and social welfare optimization in choosing the optimal form of the two-part tariff licensing contract. If $\theta$ is relatively large, then the fact that the follower turns out to be a low type makes the situation in the case of the pooling contract closer to the case of the excluding contract, but the lower royalty rate charged in the former case allows the follower to be more aggressive and produce efficiently, which will induce a more intense market competition. As a consequence, the higher expected total quantity results in higher 
social welfare. Hence, the pooling contract is preferred by society as it is consistent with the social optimally, which requires that the innovation be licensed fully disseminated and all the firms produce efficiently.

\section{Conclusions}

We have examined a Stackelberg leader's licensing behavior of an innovation when its rival holds private information about the costs after licensing occurs. The results show that the optimal licensing contract is either a excluding contract only for the low type rival being licensed by a pure royalty, or a separating contract with both type rivals being licensed by different royalty rates, which mainly depends on the possibility that the rival emerge as a low type one. This serves to verify the preference of royalty licensing by the innovator in a Stackelberg competition structure given asymmetric information and the option of a two-part tariff contract.

In addition, from the perspective of social welfare, given the possibility that the rival is a low type is relative large, social welfare in pooling contract is better than the other two forms. It demonstrates that there is still a conflict between the innovator and the whole society even under asymmetric information.

Overall, the result derived in this paper may provide a new explanation to the optimal licensing choice of the two-part tariff licensing contract when the both parties have asymmetric information about the marginal cost after licensing occurs. However, our analysis is based on the stylized set-up and the robustness still needs to be verified in more sophisticated frameworks. For example, the demand function for the product is restricted to be linear. The future work can be made with a more general demand function. This could include not only alternative functional forms, but also imagining competitors with differentiated products. In addition, in this paper, the two firms are all assumed to be fully private. What will happen if the leading firm is a mixed ownership one? Thus, the case of a mixed competition may be another direction for future research.

\section{Acknowledgements}

This research is supported by the Fundamental Research Funds for the Central Universities [Grant No. 2016XS78] and Research Base Project of Beijing Social Science Foundation [Grant No.16JDGLB023]. The usual disclaimer applies.

\section{References}

1. A. Bagchi, and A. Mukherjee, "Technology licensing in a differentiated oligopoly," International Review of Economics and Finance, vol. 29, no. 1, pp. 455-465, 2014

2. Y. W. Chen, L. F. S. Wang, S. J. Wu, and Y. P. Yang, “Technology licensing in mixed oligopoly," International Review of Economics and Finance, vol. 31, no. 338, pp. 193-204, 2014

3. N. T. Gallini, and B. D. Wright, "Technology transfer under asymmetric information," RAND Journal of Economics, vol. 21, no. 1, pp. $147-160,1990$

4. J. S. Heywood, J. Li, and G. Ye, "Per unit vs. ad valorem royalties under asymmetric information," International Journal of Industrial Organization, vol. 37, no. 1, pp. 38-46, 2014

5. Y. Li, and T. Yanagawa, "Patent licensing of Stackelberg manufacturer in a differentiated product market," International Journal of Economic Theory, vol. 7, no. 1, pp. 7-20, 2011.

6. S. Kishimoto, and S. Muto, "Fee versus royalty policy in licensing through bargaining: An application of the Nash bargaining solution," Bulletin of Economic Research, vol. 64, no.2, pp. 293-304, 2012

7. S. Poddar, and U. B. Sinha, "The role of fixed fee and royalty in patent licensing," Departmental Working Papers, vol. 121 , no. 1, pp. 53-69, 2002

8. P. W. Schmitz, "On monopolistic licensing strategies under asymmetric information," Journal of Economic Theory, vol. 106, no. 1, pp.177-189, 2002

9. D. Sen, "On the coexistence of different licensing schemes," International Review of Economics and Finance, vol. 14, no. 4, pp. 393-413, 2005

10. D. Sen, and Y. Tauman, "General licensing schemes for a cost-reducing innovation," Games and Economic Behavior, vol.59, no. 1, pp. 163-186, 2007

11. X. H. Wang, "Fee versus royalty licensing in a Cournot duopoly model," Economic Letter, vol. 60, no.1, pp. 55-62, 1998

12. X. H. Wang, "Fee versus royalty licensing in a differentiated Cournot duopoly," Journal of Economics and Business, vol. 54, no. 2, pp. 253-266, 2002

13. Q. Yan, and L. Yang, "Optimal licensing in a differentiated Bertrand market under uncertain R\&D outcomes and technology spillover," Economic Modelling (online since July 20, 2017) (http://dx.doi.org/10.1016/j.econmod.2017.06.013) 